\title{
Vladimir Shalack
}

\section{ON RELATIONAL AND FUNCTIONAL LANGUAGES}

\begin{abstract}
We prove two theorems concerning expressive power of relational and functional languages. The theorems have interesting consequences for the history of philosophy and logic.
\end{abstract}

Keywords: relational language, functional language, substantive metaphysics

\section{Introduction}

It is a quite common view that the transition to modern logic, made at the turn of the XX century, was associated with the increase in the expressive power of language through the inclusion of, among other things, judgments about relationships. It is believed that the transition to the language and the logic of relations was a natural step, since it allows an opportunity to give a more complete description of the world around us. It enriched not only the logic itself, but also the overall development of science.

This view was shared by R. Carnap, when he wrote in [1] that the restriction only to predicative sentences fatally affected areas lying outside the sphere of logic, that Russell was right explaining some of the errors of metaphysics by shortcomings of logic, and that any substantive metaphysics can be explained as based on this error, which allegedly caused a long delay in the development of physics, giving rise to substantive view of matter.

We show that, contrary to the received view, the language of properties (one-place relations) and functions is sufficient for the expression of the same mathematical and physical ideas, which are usually presented 
in terms of multi-place relations. We must conclude that, from a logical point of view, there was no need to abandon the subject-predicate language and substantive metaphysics.

\section{Basic notions}

If $S$ is a theory in a language $L$, then $A \in L$ means that the formula $A$ belongs to $L$, and $S \vdash A$ means, that the formula $A$ is provable in the theory $S$.

We say that a theory $S_{1}$ is subtheory of $S_{2}$ (in short: $S_{1} \subseteq S_{2}$ ) if and only if $L_{1} \subseteq L_{2}$ and from $S_{1} \vdash A$ it follows $S_{2} \vdash A$.

Let $S_{1}$ and $S_{2}$ be two theories in languages $L_{1}$ and $L_{2}$, respectively. A recursive function $\varphi: L_{1} \rightarrow L_{2}$ is called operation, which embeds $S_{1}$ into $S_{2}$, if and only if $\varphi$ satisfies the following condition for any $A \in L_{1}$ :

$$
S_{1} \vdash A \Longleftrightarrow S_{2} \vdash \varphi(A) .
$$

We say that a theory $S_{1}$ is embeddable in a theory $S_{2}$ if and only if there is an operation which embeds $S_{1}$ in $S_{2}$. Theories $S_{1}$ and $S_{2}$ are mutually embeddable if and only if $S_{1}$ is embeddable in $S_{2}$, and $S_{2}$ is embeddable into $S_{1}$. Relation of being mutually embeddable is reflexive, symmetric and transitive [4].

Lemma 1. Let $S_{1} \subseteq S_{2}$ and there exists a recursive function $\phi: L_{2} \rightarrow L_{1}$ which satisfies the following conditions:

(i) if $A \in L_{2}$ and $S_{2} \vdash A$, then $S_{1} \vdash \phi(A)$,

(ii) if $A \in L_{1}$ and $S_{1} \vdash \phi(A)$, then $S_{1} \vdash A$,

(iii) if $A \in L_{2}$ and $S_{2} \vdash \phi(A)$, then $S_{2} \vdash A$.

Then

1. the identity function $\iota(A)=A$ embeds $S_{1}$ in $S_{2}$,

2. the function $\phi$ embeds $S_{2}$ in $S_{1}$,

Thus, the theories $S_{1}$ and $S_{2}$ are mutually embeddable.

Proof. 1. For any $A \in L_{1}$ : if $S_{1} \vdash A$, i.e. $S_{1} \vdash \iota(A)$, then $S_{2} \vdash \iota(A)$, by $S_{1} \subseteq S_{2}$. Reversely, if $S_{2} \vdash \iota(A)$, i.e. $S_{2} \vdash A$, then $S_{1} \vdash \phi(A)$, by (i). So $S_{1} \vdash A$, by (ii).

2. For any $A \in L_{2}$ : if $S_{2} \vdash A$, then $S_{1} \vdash \phi(A)$, by (i). Reversely, if $S_{1} \vdash \phi(A)$, then $S_{2} \vdash \phi(A), S_{1} \subseteq S_{2}$. Hence $S_{2} \vdash A$, by (iii). 


\section{The main result}

Let $T_{1}$ be an axiomatic first-order theory in a language $L_{1}$ with equality such that $T_{1} \vdash \neg a=b$, for some closed terms $a$ and $b$ of $L_{1}$. Then for any $n$-ary predicate symbol $P$ of $L_{1}$, the following formulas are provable in $T_{1}$ :

$$
\begin{aligned}
\exists y((P(\boldsymbol{x}) \wedge y=a) & \vee(\neg P(\boldsymbol{x}) \wedge y=b)) \\
{[((P(\boldsymbol{x}) \wedge y=a) \vee(\neg P(\boldsymbol{x}) \wedge y=b))} & \wedge((P(\boldsymbol{x}) \wedge z=a) \\
& \vee(\neg P(\boldsymbol{x}) \wedge z=b))] \supset y=z
\end{aligned}
$$

where $\boldsymbol{x}$ is an $n$-tuple of pairwise distinct variables, and different from the variable $y$.

The above facts allow us to construct the theory $T_{2}$, obtained by expanding the language $L_{1}$ to $L_{2}$ by adding for each $n$-placed predicate symbol $P$ a new functional symbol $f_{P}$ and a new axiom

$$
\left(P\left(x_{1}, \ldots, x_{n}\right) \wedge f_{P}\left(x_{1}, \ldots, x_{n}\right)=a\right) \vee\left(\neg P\left(x_{1}, \ldots, x_{n}\right) \wedge f_{P}\left(x_{1}, \ldots, x_{n}\right)=b\right)
$$

The reason for the introduction of new functional symbols is Theorem 2.28 from Mendelson [3]. For each functional symbol $f_{P}$ the following formulas are provable in $T_{2}$ :

$$
\begin{gathered}
f_{P}\left(x_{1}, \ldots, x_{n}\right)=a \vee f_{P}\left(x_{1}, \ldots, x_{n}\right)=b \\
P\left(x_{1}, \ldots, x_{n}\right) \equiv f_{P}\left(x_{1}, \ldots, x_{n}\right)=a
\end{gathered}
$$

From Theorem 2.28 of Mendelson [3] and Theorem 42 of Kleene [2] we obtain the following two facts.

Lemma 2. $T_{1} \subseteq T_{2}$ and $T_{2}$ is a conservative extension of $T_{1}$. So for any $A \in L_{1}: T_{1} \vdash A$ iff $T_{2} \vdash A$.

Lemma 3. There exists a recursive function $\phi: L_{2} \rightarrow L_{1}$ such that:

(1) if $A \in L_{2}$ then $T_{2} \vdash A \equiv \phi(A)$,

(2) if $A \in L_{2}$ and $T_{2} \vdash A$, then $T_{1} \vdash \phi(A)$.

Lemma 4. Theories $T_{1}$ and $T_{2}$ are mutually embeddable.

Proof. Since $T_{1} \subseteq T_{2}$, it suffices to find a recursive function satisfying the conditions of Lemma 1 . We show that the mapping $\phi$ from Lemma 3 possesses the required properties. 
Indeed, by Lemma 3(2), we obtain the condition (i) of Lemma 1.

For (ii): if $A \in L_{1}$ and $T_{1} \vdash \phi(A)$, then $T_{2} \vdash \phi(A)$, by $T_{1} \subseteq T_{2}$. Hence $T_{2} \vdash A$, by Lemma 3(1). Thus $T_{1} \vdash A$, by Lemma 2 .

For (iii): if $A \in L_{2}$ and $T_{2} \vdash \phi(A)$, then $T_{2} \vdash A$, by Lemma 3(2). $\dashv$

Define the language $L_{3}$, which is obtained from the language $L_{2}$ by deleting all predicate symbols other than the symbol of equality. Next, we define the following function $\alpha: L_{2} \rightarrow L_{3}$ such that:

- $\alpha\left(t_{1}=t_{2}\right)=\left\ulcorner t_{1}=t_{2}\right\urcorner$,

- $\alpha\left(P\left(t_{1}, \ldots, t_{n}\right)\right)=\left\ulcorner f_{P}\left(t_{1}, \ldots, t_{n}\right)=a\right\urcorner$, and $P$ is different from ' $=$ ',

- $\alpha(\neg A)=\ulcorner\neg \alpha(A)\urcorner$,

- $\alpha(A \circ B)=\ulcorner(\alpha(A) \circ \alpha(B))\urcorner$, where $\circ \in\{\wedge, \vee, \supset, \equiv\}$

- $\alpha(Q x B)=\ulcorner Q x \alpha(B)\urcorner$, where $Q \in\{\forall, \exists\}$.

We define the theory $T_{3}$ in the language $L_{3}$ by the following condition:

$A$ is a non-logical axiom of the theory $T_{3}$ if and only if $A=\alpha(B)$ for some a non-logical axiom $B$ of $T_{2}$.

LEMMA $5 . T_{3} \subseteq T_{2}$.

Proof. By the definitions, $L_{3} \subseteq L_{2}$ and for any non-logical axiom $A$ of $T_{3}$ there is a non-logical axiom $B$ of $T_{2}$ such that $A=\alpha(B)$. Moreover, since $T_{2} \vdash(\ddagger)$, by the definition of $\alpha$ and the Equivalence Theorem we have that

$$
T_{2} \vdash B \equiv \alpha(B) \text { and } T_{2} \vdash \alpha(B) .
$$

From this fact it follows that any proof in the theory $T_{3}$ at the same time is a proof in the theory $T_{2}$ and consequently $T_{3} \subseteq T_{2}$.

Lemma 6. (1) The identity function $\iota(A)=A$ embeds $T_{3}$ in $T_{2}$.

(2) The function $\alpha$ embeds $T_{2}$ in $T_{3}$.

Thus, the theories $T_{2}$ and $T_{3}$ are mutually embeddable.

Proof. Since $T_{3} \subseteq T_{2}$, we need to show that $\alpha$ is a recursive function satisfying the conditions of Lemma 1.

For (i): By induction on the construction of a proof of a formula $A$ in $T_{2}$, we demonstrate that formula $\alpha(A)$ is provable in the theory $T_{3}$.

If $A$ is a logical axiom of $T_{2}$, then $\alpha(A)$ is also a logical axiom and it is provable in $T_{3}$. If $A$ is a non-logical axiom of $T_{2}$, then $\alpha(A)$ is a non-logical axiom of $T_{3}$. 
Suppose that a formula $A$ is obtained by the rule of modus ponens from two previous formulas $B$ and $\ulcorner B \supset A\urcorner$. By assumption, formulas $\alpha(B)$ and $\alpha(B \supset A)$ are provable in $T_{3}$. Hence the formula $\alpha(A)$ is also provable in $T_{3}$.

Suppose that a formula $A=\ulcorner\forall x B\urcorner$ and $A$ is obtained by the rule of generalization from $B$. By assumption, the formula $\alpha(B)$ is provable in $T_{3}$. Hence, the same holds good for $\ulcorner\forall x \alpha(B)\urcorner$. Since $\ulcorner\forall x \alpha(B)\urcorner=$ $\ulcorner\alpha(\forall x B)\urcorner$ it follows that the formula $\ulcorner\alpha(\forall x B)\urcorner$ is provable in $T_{3}$.

Thus, we have shown that from $T_{2} \vdash A$ it follows $T_{3} \vdash \alpha(A)$.

For (ii): If $A \in L_{3}$ and $T_{3} \vdash \alpha(A)$, then $T_{3} \vdash A$, since $\alpha(A)=A$, by the definition of $\alpha$.

For (iii): Suppose that $A \in L_{2}$ and $T_{2} \vdash \alpha(A)$. Since $T_{2} \vdash(\ddagger)$, by the Equivalence Theorem, we have that $T_{2} \vdash A \equiv \alpha(A)$. Hence $T_{2} \vdash A$.

The next theorem follows from lemmas 4 and 6 .

Theorem 1. Let $T$ be a first-order theory with equality such that $T_{1} \vdash$ $\neg a=b$, for some closed terms $a$ and $b$. Then there exists a firstorder theory $T^{\prime}$ such that $T$ and $T^{\prime}$ are mutually embeddable, and $T^{\prime}$ is formulated in a language with functional symbols, and only the equality predicate.

We can go further and show that the logical predicate of equality is not necessary and can be replaced by a special one-place predicate.

Let the theory $T_{4}$ be obtained by expanding the language of the theory $T_{3}$ with the new monadic predicate symbol ' $H$ ' and addition of the following new axiom

$$
H(x) \equiv x=a
$$

Notice that, by the definition, $T_{3} \subseteq T_{4}$.

Lemma 7. The following formula

$$
H\left(f_{=}\left(x_{1}, x_{2}\right)\right) \equiv x_{1}=x_{2}
$$

is provable in the theory $T_{4}$.

Proof. Since $T_{2} \vdash(\ddagger)$, so $T_{2} \vdash x_{1}=x_{2} \equiv f_{=}\left(x_{1}, x_{2}\right)=a$. By the definition of $\alpha$ and Lemma 6(2), we have that $T_{3} \vdash x_{1}=x_{2} \equiv$ $f_{=}\left(x_{1}, x_{2}\right)=a$. Hence also $T_{4} \vdash x_{1}=x_{2} \equiv f_{=}\left(x_{1}, x_{2}\right)=a$, since $T_{3} \subseteq$ $T_{4}$. Now notice that $T_{4} \vdash H\left(f_{=}\left(x_{1}, x_{2}\right)\right) \equiv f_{=}\left(x_{1}, x_{2}\right)=a$, because $\forall x(H(x) \equiv x=a)$ is an axiom of $T_{4}$. Thus, $T_{4} \vdash H\left(f_{=}\left(x_{1}, x_{2}\right)\right) \equiv x_{1}=$ $x_{2}$. 
Lemma 8. (1) The identity function $\iota(A)=A$ embeds $T_{3}$ in $T_{4}$.

(2) There is a function $\psi: L_{4} \rightarrow L_{3}$ which embeds $T_{4}$ in $T_{3}$.

Thus, the theories $T_{3}$ and $T_{4}$ are mutually embeddable.

Proof. Since $T_{3} \subseteq T_{4}$, it is enough to find a recursive function $\psi: L_{4} \rightarrow$ $L_{3}$, satisfying the conditions of Lemma 1 . We define $\psi$ as follows:

- $\psi(H(t))=\ulcorner t=a\urcorner$,

- $\psi\left(t_{1}=t_{2}\right)=\left\ulcorner t_{1}=t_{2}\right\urcorner$,

- $\psi(\neg A)=\ulcorner\neg \psi(A)\urcorner$,

- $\psi(A \circ B)=\ulcorner(\psi(A) \circ \psi(B))\urcorner$, where $\circ \in\{\wedge, \vee, \supset, \equiv\}$,

- $\psi(Q x B)=\ulcorner Q x \psi(B)\urcorner$, where $Q \in\{\forall, \exists\}$.

For (i): By induction on the construction of a proof of a formula $A$ in $T_{4}$ we demonstrate that formula $\psi(A)$ is provable in the theory $T_{3}$.

If $A$ is a logical axiom of $T_{4}$, then $\psi(A)$ is also a logical axiom and it is provable in $T_{3}$.

If $A$ is the axiom ' $H(x) \equiv x=a$ ', then $\psi(A)$ is ' $x=a \equiv x=a$ ', so $T_{3} \vdash \psi(A)$.

If $A$ is a non-logical axiom of $T_{4}$ which is a non-logical axiom of $T_{3}$, then $\psi(A)=A$, because $A$ contains no occurrences of ' $H$ '. Therefore $T_{3} \vdash \psi(A)$.

For the rule of modus ponens and the rule of generalization we apply in the standard way the definition of the function $\psi$. Thus, we have shown that from $T_{4} \vdash A$ it follows $T_{3} \vdash \psi(A)$.

For (ii): Suppose that $A \in L_{3}$ and $T_{3} \vdash \psi(A)$. Then $\psi(A)=A$, because $A$ contains no occurrences of ' $H$ '. Therefore, $T_{3} \vdash A$.

For (iii): Suppose that $A \in L_{4}$ and $T_{4} \vdash \psi(A)$. Since $\forall x(H(x) \equiv$ $x=a)^{\prime}$ ' is an axiom of $T_{4}$, so $T_{4} \vdash \psi(A) \equiv A$, from the Equivalence Theorem. Thus, $T_{4} \vdash A$.

Let the language $L_{5}$ be obtained by deleting symbol of equality from the language $L_{4}$. We define the following function $\beta: L_{4} \rightarrow L_{5}$ :

- $\beta(H(t))=\ulcorner H(t)\urcorner$,

- $\beta\left(t_{1}=t_{2}\right)=\left\ulcorner H\left(f_{=}\left(t_{1}, t_{2}\right)\right)\right\urcorner$,

- $\beta(\neg A)=\ulcorner\neg \beta(A)\urcorner$,

- $\beta(A \circ B)=\ulcorner(\beta(A) \circ \beta(B))\urcorner$, where $\circ \in\{\wedge, \vee, \supset, \equiv\}$,

- $\beta(Q x B)=\ulcorner Q x \beta(B))\urcorner$, where $Q \in\{\forall, \exists\}$. 
We define the theory $T_{5}$ as follows:

$A$ is a non-logical axiom of the theory $T_{5}$ if and only if $A=\alpha(B)$ for some $B$ which either is an axiom of equality or is a non-logical axiom of $T_{4}$.

Lemma 9. $T_{5} \subseteq T_{4}$.

Proof. If $A$ is an axiom of $T_{5}$, then $A=\beta(B)$, for some $B$ which is an axiom of equality or is a non-logical axiom of $T_{4}$. By Lemma 7 , the definition of $\beta$ and the Equivalence Theorem, we have that $T_{4} \vdash B \equiv$ $\beta(B)$ and $T_{4} \vdash \beta(B)$. Therefore any proof in the theory $T_{5}$ at the same time is the proof in $T_{4}$ and consequently $T_{5} \subseteq T_{4}$.

Lemma 10. (1) The identity function $\iota(A)=A$ embeds $T_{5}$ in $T_{4}$.

(2) The function $\beta: L_{4} \rightarrow L_{5}$ embeds $T_{4}$ in $T_{5}$.

Thus, the theories $T_{4}$ and $T_{5}$ are mutually embeddable.

Proof. Since $T_{5} \subseteq T_{4}$, we need to show thet $\beta$ is a recursive function satisfying the conditions of Lemma 1 .

For (i): By induction on the construction of a proof of a formula $A$ in $T_{4}$ we demonstrate that $T_{5} \vdash \beta(A)$.

If $A$ is a propositional or quantifier axiom of $T_{4}$, then $\beta(A)$ is a logical axiom of $T_{5}$, and therefore $T_{5} \vdash \beta(A)$.

If $A$ is an axiom of equality of $T_{4}$ or $A$ is a non-logical axiom of $T_{4}$, then $\beta(A)$ is a non-logical axiom of $T_{5}$ and therefore $T_{5} \vdash \beta(A)$.

As in the case of $\psi$, for the rule of modus ponens and the rule of generalization we apply in the standard way the definition of the function $\beta$. Thus, we have shown that from $T_{4} \vdash A$ follows $T_{5} \vdash \beta(A)$.

For (ii): If $A \in L_{5}$ and $T_{5} \vdash \beta(A)$, to $T_{5} \vdash A$, since $\beta(A)=A$, by the definition of $\beta$.

For (iii): Suppose that $A \in L_{4}$ and $T_{4} \vdash \beta(A)$. By Lemma 7 and the Equivalence Theorem, we have that $T_{4} \vdash A \equiv \beta(A)$. So $T_{4} \vdash A$.

The following theorem is a consequence of Theorem 1 and lemmas 8 and 10 .

TheOrem 2. Let $T$ be a first-order theory with equality such that $T_{1} \vdash$ $\neg a=b$, for some closed terms $a$ and $b$. Then there exists a first-order theory $T^{\prime}$ such that $T$ and $T^{\prime}$ are mutually embeddable and the language of $T^{\prime}$ contains only functional symbols, and a single one-place predicate. 


\section{Concluding remarks}

The proofs of the theorems in Section 3 are not complicated, and because of that, their content seems to be trivial. However, from a philosophical point of view, they are interesting, because they refute some widespread views. The ontology of things, properties, and functions is not worse than the ontology of things and relations, which de facto has become a model for the presentation of scientific ideas.

Moreover, it appears that we can also delete properties from our model. The ontology of objects and functions is also universal in its expressive power. The relation of equality, which is included in the language, is purely logical and says if two terms denote the same object or not.

For the philosophically oriented logician it is obvious that the replacement of one ontology by another is not a purely formal trick, but brings a completely different view of the surrounding world, leads us to use new heuristics in the construction of scientific theories.

\section{References}

[1] Carnap, R., "Die alte und die neue Logik", Erkenntnis, Bd. I, Leipzig, 1930-1931, p. 12-26.

[2] Kleene, S. C., Introduction to Metamathematics, Elsevier Science Ltd, 1952.

[3] Mendelson, E., Introduction to Mathematical Logic, D. Van Nostrand Co. Inc., New York, 1997 (fourth edition).

[4] Smirnov, V.A., Logicheskie metody analiza nauchnogo znanija, Nauka, Moskva, 1987.

Vladimir Shalack

Section of Logic

Institute of Philosophy

Russian Academy of Sciences

Volkhonka 14

119991 Moscow, Russia

shalack@mail.ru 